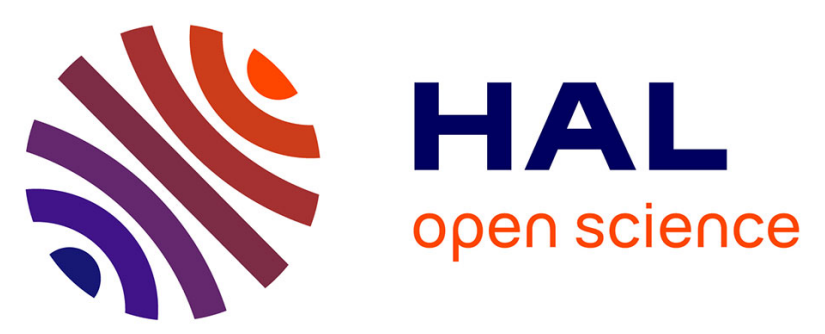

\title{
Unexpected value of the transition pressure in ionic layered BaFI compound observed by Raman scattering
}

F. Decremps, M. Gauthier, J.-C. Chervin, M. Fischer, A. Polian

\section{To cite this version:}

F. Decremps, M. Gauthier, J.-C. Chervin, M. Fischer, A. Polian. Unexpected value of the transition pressure in ionic layered BaFI compound observed by Raman scattering. Physical Review B: Condensed Matter and Materials Physics (1998-2015), 2002, 66 (2), 10.1103/PhysRevB.66.024115. hal-01921264

\section{HAL Id: hal-01921264 https://hal.sorbonne-universite.fr/hal-01921264}

Submitted on 13 Nov 2018

HAL is a multi-disciplinary open access archive for the deposit and dissemination of scientific research documents, whether they are published or not. The documents may come from teaching and research institutions in France or abroad, or from public or private research centers.
L'archive ouverte pluridisciplinaire $\mathbf{H A L}$, est destinée au dépôt et à la diffusion de documents scientifiques de niveau recherche, publiés ou non, émanant des établissements d'enseignement et de recherche français ou étrangers, des laboratoires publics ou privés. 


\title{
Unexpected value of the transition pressure in ionic layered BaFI compound observed by Raman scattering
}

\author{
F. Decremps, M. Gauthier, J.-C. Chervin, M. Fischer and A. Polian \\ Laboratoire de Physique des Milieux Condensés, CNRS-UMR 7602, \\ Université Pierre \& Marie Curie, Tour 13, B7r, \\ 4, place Jussieu, 75252 Paris CEDEX 05, France*
}

(Dated: June 4, 2002)

\begin{abstract}
The high pressure behavior of optical phonons in layered BaFI has been studied at ambient temperature using Raman spectroscopy up to $61 \mathrm{GPa}$. The pressure dependence of the zone-center phonons $\mathrm{B}_{1 g}, \mathrm{E}_{g}$, and $\mathrm{A}_{1 g}$ was measured in the tetragonal structure up to the phase transition near $55 \mathrm{GPa}$. The Grüneisen parameter of the low frequency $\mathrm{A}_{1 g}$ mode has a very large variation between 0 and $10 \mathrm{GPa}$. This evolution is attributed to a gradual layer-nonlayer transformation of the structure under pressure. For ionic compounds, and with respect to the classical hard-sphere model, phase transition tends to occur at about the same effective hard-sphere packing fraction. Consequently, the phase transition in BaFI could be thought to occur at lower pressure than in $\mathrm{BaFCl}$ or $\mathrm{BaFBr}$, in contradiction with our experimental data. Using thermodynamics arguments, the unexpected increase of the phase transition pressure with increasing halogen size in BaFX $(\mathrm{X}=\mathrm{Cl}, \mathrm{Br}$ or $\mathrm{I})$ is attributed to the bi-dimensional characteristic of the structure which is shown to expand the lattice volume and to decrease the internal energy by a polarization contribution $\mathrm{U}_{\text {pol }}$.
\end{abstract}

PACS numbers: 78.30.-j, 62.50.+p, 61.50.Ks

\section{INTRODUCTION}

Layered materials have generated considerable interest since recent investigations have resulted in many remarkable achievements in semiconductors[1], lubricants[2], superconductors[3] or intercalation storages like graphyne [4] or fluoro-halide (matlockite) compounds [5]. The generic chemical formula of matlockite family compounds is MFX where $\mathrm{M}$ is the metallic ion $\mathrm{Ca}, \mathrm{Sr}, \mathrm{Ba}$, $\mathrm{Pb}$ or $\mathrm{Eu}$, and $\mathrm{X}$ the halogen $\mathrm{Cl}, \mathrm{Br}$ or I. The tetragonal structure of the fluoro-halide crystals MFX has been shown to exhibit anisotropic compressibilities[6], directly related to the observed macroscopic layered properties. Elastic anisotropy of typical layered crystals is known to be related to the existence of two distinct kinds of interatomic bonding: a covalent (or metallic) one inside the layers and van der Waals type forces between the sheets. In the case of MFX compounds, the layer-like conformation is quite surprising since these crystals are strongly ionic. In Ref. [6], based on X-ray diffraction results, the stability of ionic MFX crystals in a layered structure is simply explained in terms of anisotropic Coulomb forces due to the asymmetric position of large polarizable halogen $X$ atoms in their $M_{5}$ metallic pyramid cage (see Fig. 1). However, these assumptions still need to be confirmed by measurements carried out with a short-range order probe. The first part of the present work is devoted to the high pressure Raman scattering study of BaFI, one of the most layered fluoro-halides crystal. This compound crystallizes at ambient conditions in the tetragonal structure $\mathrm{P} 4 / \mathrm{nmm}\left(\mathrm{D}_{4 h}^{7}\right)$. At the $\Gamma$ point of the Brillouin

*Electronic address: frederic.decremps@pmc.jussieu.fr zone, group theory predicts the existence of the following optical modes: $\Gamma_{\text {opt }}=2 \mathrm{~A}_{1 g}+\mathrm{B}_{1 g}+3 \mathrm{E}_{g}+3 \mathrm{~A}_{2 u}+3 \mathrm{E}_{u} . \mathrm{A}_{1 g}$, $\mathrm{B}_{1 g}$, and $\mathrm{E}_{g}$ modes are Raman active. The mode assignment of $\mathrm{BaFCl}, \mathrm{BaFBr}, \mathrm{BaFI}, \mathrm{PbFCl}, \mathrm{PbFBr}$ and $\mathrm{SrFCl}$ at ambient conditions have been well established in the literature [7-9]. The atomic displacement patterns related to these modes have been described in Ref. [10]. The mode labelled $\mathrm{A}_{1 g}(1)$ corresponds to the halogens motion along the $c$ axis inside the $M_{5}$ pyramid whereas the other modes are related to the metallic tetrahedra breathing along $c\left(\mathrm{~A}_{1 g}(2)\right)$, the fluor motion along $c$ inside the $M_{4}$ tetrahedra $\left(\mathrm{B}_{1 g}\right)$ and atoms motion along the $a$ axis $\left(\mathrm{E}_{g}(1,2,3)\right)$.

We also determined for the first time the transition pressure of BaFI and the second part of the discussion is devoted to the transition pressure trend with halogen size in ionic-layered compounds. Actually, large $X$ halogen atoms size may induce chemical pressure due to steric effect, which has been experimentally confirmed for most ionic crystals. For BaFX compounds, because the $\mathrm{Cl}$ ion radius $\left(\mathrm{r}_{C l}=1.81 \AA\right)$ is smaller than the $\mathrm{Br}$ one $\left(\mathrm{r}_{B r}=1.96 \AA\right), \mathrm{BaFCl}$ at high pressure may be considered as the analogous of $\mathrm{BaFBr}$ at ambient pressure[11]. Thus, one should expect a transition pressure for $\mathrm{BaFBr}$ lower than for BaFCl. This conclusion is in disagreement with experimental results, where structural phase transition have been observed to occur at pressures near 21 and $27 \mathrm{GPa}$ for $\mathrm{BaFCl}$ and $\mathrm{BaFBr}$ respectively[6, 11-13]. We confirm this unexpected result in the present work, where the phase transition of BaFI is shown to occur at pressure far above $27 \mathrm{GPa}$. 


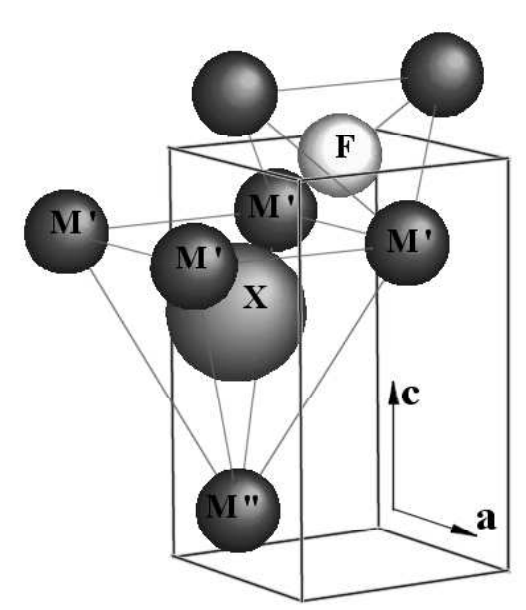

FIG. 1: Polyhedral representation of the matlockite structure. Contrary to the fluor inside the $M_{4}$ tetrahedron, the halogen $X$ in the $M_{5}$ pyramid has an asymmetric coordination: $X$ is equidistant from the four basal plane $M^{\prime}$ cations (distance $\left.M^{\prime} X\right)$ but at a different distance from the $M$ " apex cation (distance $M " X)$ ). For example, in BaFI, $d_{B a^{\prime} I}=3.582 \AA$ while $d_{B a "}{ }=3.844 \AA$ ) .

\section{EXPERIMENTS AND RESULTS}

BaFI crystals were prepared by chemical reaction of $\mathrm{BaF}_{2}$ and $\mathrm{BaI}_{2}[14]$. For the high pressure Raman experiments, the BaFI sample was cut as platelet from a colorless and transparent single-crystal. The sample was about 30x20 $\mu \mathrm{m}^{2}$ in surface and $20 \mu \mathrm{m}$ thick. Because BaFI is a layered compound, the faces perpendicular to the $c$-axis are plane, parallel and as polished. The high pressure cell was a membrane diamond anvil cell (DAC) [15]. A stainless steel gasket was preindented to a thickness of $40 \mu \mathrm{m}$ and a $150 \mu \mathrm{m}$ diameter hole was drilled in the center by spark-erosion. Nitrogen was cryogenically loaded inside the DAC and used as a pressure transmitting medium. Pressure was systematically measured before and after each Raman spectrum using the fluorescence emission of a ruby sphere [16] placed close to the sample into the gasket hole. The accuracy is better than $2 \mathrm{GPa}$ at the maximum pressure reached (61 GPa). Raman spectra were excited with the $514.5 \mathrm{~nm}$ line of an $\mathrm{Ar}^{+}$laser focused down to $5 \mu \mathrm{m}$ with a power level of about $50 \mathrm{~mW}$ at the entrance of the DAC. The scattered light was analyzed with a Dilor XY triple spectrometer and a liquid-nitrogen-cooled CCD multichannel detector. All spectra were recorded in the backscattering geometry with unpolarized light. The Raman frequencies were determined from a computer fit of the peaks with a Lorentzian profile. The accuracy is better than $1 \mathrm{~cm}^{-1}$.

Before performing the high pressure experiment, we examined the zone-center optical phonons of tetragonal BaFI at ambient conditions. We employed a large single crystal from which the micrometric sample used in the high pressure experiments was extracted. With incident radiation parallel to the $c$-axis, only $\mathrm{A}_{1 g}$ and $\mathrm{B}_{1 g}$ modes

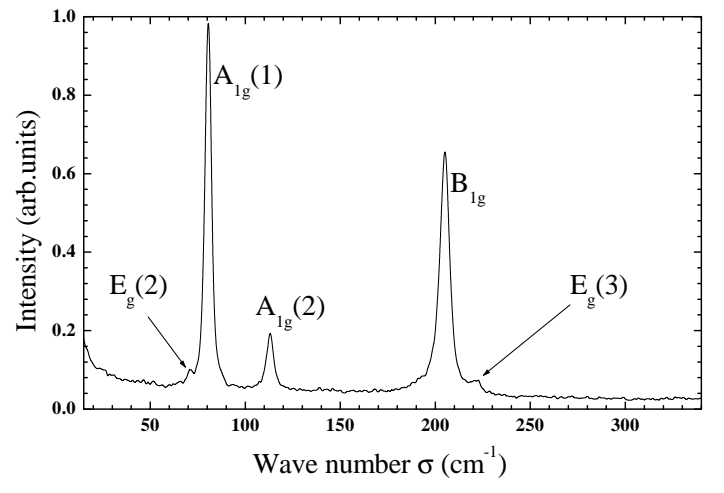

FIG. 2: Raman spectra of BaFI at ambient conditions.

TABLE I: Raman-active BaFI $\Gamma$-point phonon frequencies (in $\mathrm{cm}^{-1}$ ) at ambient conditions. The value of $\mathrm{E}_{g}(1)$ frequency (superscript $\sharp$ ) has not been measured but estimated from a comparison with $\mathrm{BaFCl}$ and $\mathrm{BaFBr}$ modes.

\begin{tabular}{cccc}
\hline \hline Mode & this work & Ref. [7] & Ref. [17] \\
\hline $\mathrm{B}_{1 g}$ & 205 & 203 & 198 \\
$\mathrm{~A}_{1 g}(1)$ & 81 & - & - \\
$\mathrm{A}_{1 g}(2)$ & 113 & - & - \\
$\mathrm{E}_{g}(1)$ & $\left(40^{\sharp}\right)$ & - & - \\
$\mathrm{E}_{g}(2)$ & 74 & - & - \\
$\mathrm{E}_{g}(3)$ & 221 & 219 & 244 \\
\hline \hline
\end{tabular}

are allowed and clearly seen in the spectra (Fig. 2). However, because of defects, two of the three $\mathrm{E}_{1 g}$ modes could also be observed in the spectra. The third $\mathrm{E}_{1 g}$ peak (labelled $\left.\mathrm{E}_{1 g}(1)\right)$ could not be seen in the present work, neither with incident light parallel nor perpendicular to $c$. A simple comparison with phonon frequency of the same mode in $\mathrm{BaFCl}$ and $\mathrm{BaFBr}$ gives $\mathrm{E}_{1 g}(1)$ around $40 \mathrm{~cm}^{-1}$ for BaFI. The frequency assignment and a comparison with previous works are given in Table I.

The high pressure experiment was carried out with incident light parallel to the tetragonal $c$-axis. The $\mathrm{B}_{1 g}$, $\mathrm{E}_{g}(3), \mathrm{A}_{1 g}(1)$ and $\mathrm{A}_{1 g}(2)$ modes have been followed and the pressure dependence of their frequencies are shown in Fig. 3. The frequency of $\mathrm{B}_{1 g}, \mathrm{E}_{g}(3)$ and $\mathrm{A}_{1 g}(2)$ modes increases with pressure and no anomaly occurs up to the transition pressure. The $\mathrm{A}_{1 g}(1)$ intensity gradually weakens under pressure up to $30 \mathrm{GPa}$ where the corresponding peak could not be seen anymore. Moreover, at low pressure, the frequency variation of this mode is considerable compared with the other ones. The significance of this observation will be addressed in the discussion.

At around $55 \mathrm{GPa}$, the vanishing of $\mathrm{E}_{g}(3)$, the appearance of new peaks and the clear increase of the $\mathrm{A}_{1 g}(2)$ mode intensity reflect the onset of a structural transformation. These effects are enhanced after 24 hours of relaxation at $61 \mathrm{GPa}$ as shown in Fig. 4. 


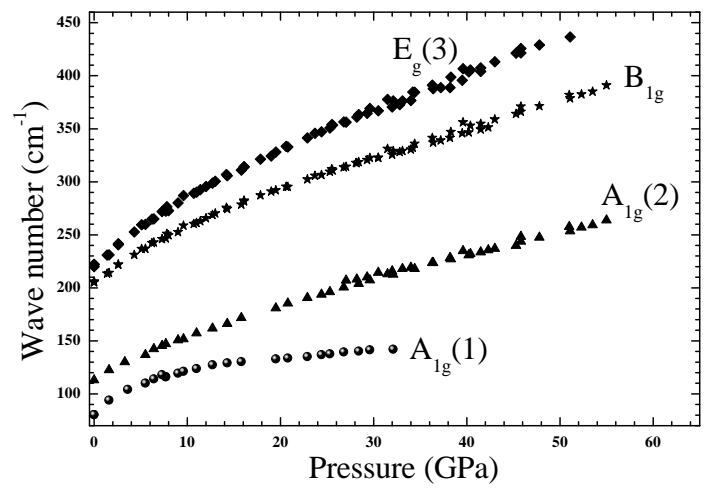

FIG. 3: Pressure dependence of the observed optical phonons in the tetragonal structure.

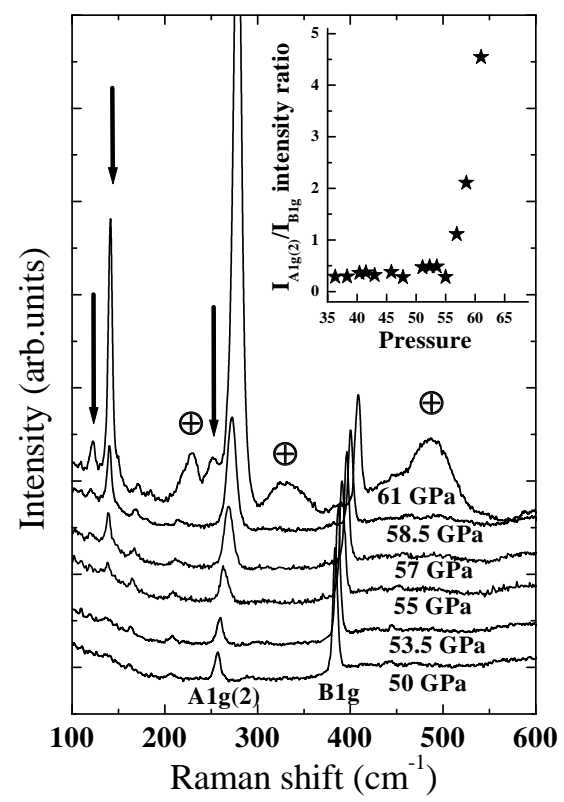

FIG. 4: Raman spectra of single-crystalline BaFI under different hydrostatic pressures in the neighborhood of the transition pressure. The spectra are shifted upward for clarity. The upper spectrum has been recorded during $2 \times 45 \mathrm{mn}(2 \times 5 \mathrm{mn}$ for the other spectra) and after 24 hours of relaxation at 61 GPa. The symbols $\oplus$ and the arrows point out nitrogen Raman modes and the new Raman peaks of the high pressure phase respectively. (Inset) Pressure dependence of the intensity ratio between the $\mathrm{A}_{1 g}(2)$ and the $\mathrm{B}_{1 g}$ peaks.

\section{DISCUSSION}

\section{A. BaFI layered character under pressure}

The mode Grüneisen parameters $\gamma_{i}$ defined as $\gamma_{i}=$ $-\left(\frac{d \ln \nu_{i}}{d \ln V}\right)_{P}$ have been calculated at different pressures. The isothermal Birch-Murnaghan equation of state was used to determine the pressure dependence of the unit cell volume with $\mathrm{B}_{0}=36 \mathrm{GPa}$ and $\mathrm{B}^{\prime}=6[6]$. Figure 5 shows a clear difference between the $\mathrm{A}_{1 g}(1)$ and the other modes. The Grüneisen parameter $\gamma$ of $\mathrm{A}_{1 g}(1)$ varies strongly between 0 and $\sim 10 \mathrm{GPa}$ which is characteristic of inter-layers cohesive forces evolution. Above 20 GPa, $\gamma\left(A_{1 g}(1)\right)$ remains around 1 , revealing a strengthening of the initial weak forces along the $c$ axis. The atomic motion related to the $\mathrm{A}_{1 g}(1)$ optic mode is also shown in figure 5 . Thus, the present data suggest that (i) the weak interactions inside the fluoride halides MFX compounds are localized between the XX double layers and (ii) the layered character disappears under pressure.

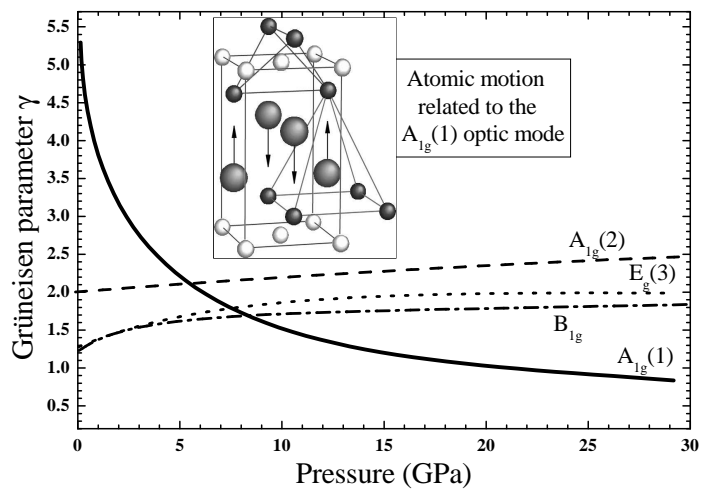

FIG. 5: Pressure dependence of the modes Grüneisen parameters $\gamma$ (left). The atomic motion related to the $\mathrm{A}_{1 g}(1)$ optic mode is also illustrated (inset).

These results are in agreement with the assumptions made in Ref [6]. Briefly, the large polarizability and asymmetrical coordination scheme of the halogen atom have been shown to create large static dipoles on the anions which stabilize the structure in a layer-like configuration. Under pressure the weak bonds are primarily affected. Therefore, the halogen is expected to move to a symmetric configuration, canceling the polarization of the $\mathrm{X}$ halogen. The pressure variation of the BaFX $\frac{c}{a}$ ratio (determined by X-ray diffraction) is significant: while its value is pressure independent for the quasi threedimensional $\mathrm{BaFCl}$ compound $\left(\frac{c}{a}=1.64\right)$, the strong decrease from ambient pressure $\left(\frac{c}{a}=1.71\right)$ to about $15 \mathrm{GPa}$ $\left(\frac{c}{a}=1.64\right)$ for BaFI is consistent with the present interpretation of a gradual layer-nonlayer transformation.

\section{B. Transition pressure}

Chemical substitution is often used to simulate pressure behavior. In BaFX family, the larger the ionic radius of the $\mathrm{X}$ halogen is, the larger the local compactness (defined as the ratio between the $\mathrm{X}$ anion volume and that of the $\mathrm{M}_{5}$ pyramid volume, see figure 1) is. In that sense, $\mathrm{BaFI}$ at ambient pressure could be thought as the analogous of $\mathrm{BaFCl}$ or $\mathrm{BaFBr}$ at high pressure. Consequently, the phase transition of BaFI should be expected at lower pressure than in $\mathrm{BaFBr}$ or $\mathrm{BaFCl}$. Experimentally and contrary to this simple representation, the tetragonal structure of BaFI appears to be extremely stable under pressure with $\mathrm{P}_{T}=55 \mathrm{GPa}$. More generally, 
it is the behavior of the whole BaFX family which contradicts the "classical" expectation since the transition pressure of $\mathrm{BaFCl}, \mathrm{BaFBr}$ and $\mathrm{BaFI}$ are 21, 27 and 55 GPa respectively. Thermodynamics arguments can however be used to understand the experimentally observed elevation of the phase transition pressure with increasing layered character in BaFX. Using the intuitive polarizable halogen model, effect of the bi-dimensional structure on the unit cell volume and the internal energy of the crystal will be considered first.

\section{Effect of the layer character on the unit cell volume.}

In classical ionic structure, the value of the lattice parameters is only related to a simple steric effect. For example, in tetragonal structure the length change of $a$ and $c$ with ions substitution is expected to be homothetic with a constant value of $\frac{c}{a}$. On the other hand, for bidimensional compounds with layers perpendicular to the $c$ axis as MFX, while the length of $a$ is actually due to a steric effect, the weak bonds between the halogen planes increase the lattice parameter $c$ leading to a layer-like conformation with a higher volume and higher $\frac{c}{a}$ ratio as compared to the corresponding three-dimensional structure where only steric effect occur, see Table II.

TABLE II: Crystal data for BaFX compounds. V, a and c are the experimental lattice volume and lattice parameters respectively. $\mathrm{c}^{\text {iso }}$ and $\mathrm{V}^{\text {iso }}$ have been calculated using the experimental $\frac{c}{a}$ of the quasi three-dimensional crystal BaFCl. The difference $\Delta \mathrm{V}=\mathrm{V}-\mathrm{V}^{\text {iso }}$ pictures the lattice expansion induced by the layer-like conformation.

\begin{tabular}{cccccccc}
\hline \hline Crystal & $\mathrm{a}(\stackrel{\circ}{ })$ & $\mathrm{c}\left(\AA^{\circ}\right)$ & $\frac{c}{a}$ & $\mathrm{~V}\left(\AA^{3}\right)$ & $\mathrm{c}^{\text {iso }}(\AA)$ & $\mathrm{V}^{\text {iso }}\left(\AA^{3}\right)$ & $\Delta \mathrm{V}\left(\AA^{3}\right)$ \\
\hline $\mathrm{BaFCl}$ & 4.394 & 7.225 & 1.64 & 139.5 & 7.225 & 139.5 & 0 \\
$\mathrm{BaFBr}$ & 4.508 & 7.441 & 1.65 & 150.9 & 7.385 & 149.7 & 1.2 \\
$\mathrm{BaFI}$ & 4.654 & 7.962 & 1.71 & 172.5 & 7.632 & 165.3 & 7.2 \\
\hline \hline
\end{tabular}

\section{Effect of the layer character on the crystal energy.}

Because the electronic polarizability $\alpha$ is directly proportional to the atomic size, large size halogen anions (like iodine) are highly polarizable. Combined with the halogen anisotropic coordination $\left(d_{M^{\prime} X} \neq d_{M^{\prime} X}\right.$, see Fig. 1), these characteristics create a large static dipole on the halogen. The corresponding local electric field $\vec{E}$ gives an extra cohesive energy term $U_{\text {pol }}$ to the threedimensional lattice energy with: $U_{p o l}=-\frac{1}{2} N \alpha \vec{E}^{2}(N$ is the number of anions). Thus, a layered structure for ionic crystals with large halogen atoms appears to be the most favorable framework in terms of stability.

\section{Thermodynamics of the phase transition.}

Thermodynamically, the equilibrium condition between two phases at temperature $T$ is given by: $P_{T}=$ $-\left.\frac{\partial F}{\partial V}\right|_{T}$, where $\mathrm{F}$ is the Helmholtz free energy defined by $F=U-T S$ ( $U$ and $S$ are the internal energy and the entropy respectively). The entropy change at the transition has been ignored [18]. Thus, the pressure transition can be simply defined as the slope of the common tangent to both low and high pressure phases $U(V)$ curves. In Figure 6 , the energy curve corresponding to the layered MFX structure is shifted with respect to a three-dimensional structure to lower energy (due to the $\mathrm{U}_{p o l}$ term) and higher volume (by $\Delta \mathrm{V}$ ). As the two-dimensional character increases, the slope of the common tangent to the tetragonal and the high pressure phase energy curves increases, and therefore the phase transition pressure increases.

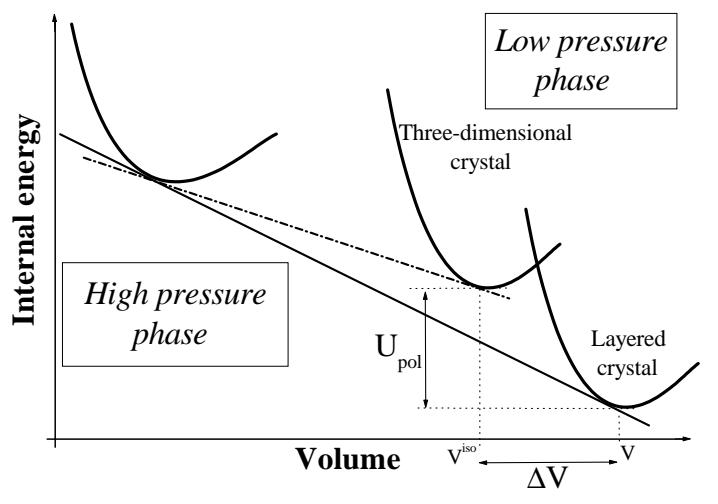

FIG. 6: Energy volume curves for two-and three-dimensional MFX crystal. The offset of the high pressure phase with respect to the tetragonal curves determines the phase transition pressure. For crystals with highly polarizable halogen atoms like BaFI, the large value of $\frac{\partial U_{p o l}}{\partial \Delta V}$ increases the stability pressure range.

\section{CONCLUSION}

In this paper, we have examined the pressure variation of the optical phonons frequencies in BaFI. The results suggest a gradual layer to three dimensional evolution of the tetragonal structure under pressure. Moreover, the ambient pressure phase of BaFI appears to be extremely stable under pressure with a transition pressure of 55 GPa. This result shows that, doped with rare-earth ions, $\mathrm{BaFI}$ is potentially of most interest for technological applications as in-situ pressure gauge for experiments with diamond anvil cell [19]. In the high pressure phase, three new peaks are observed which is not contradictory with the proposed $\mathrm{P} 22_{1} / \mathrm{m}$ space group [13]. We also attempted to understand why $\mathrm{BaFI}$ is more stable than $\mathrm{BaFCl}$ or $\mathrm{BaFBr}$ under pressure, in disagreement with the intuitive hard-sphere model. Taking into account the contribution 
of the polarization term due to the highly polarizable halogen atoms, a thermodynamic analysis allows to answer to this question. Finally, and from a more general point of view, the present study may also contribute to a better understanding of the chemical substitution effect origin on the critical temperature $\mathrm{T}_{c}$ of layered superconducting systems. As a matter of fact, the response of superconductors to pressure is important since a large pressure derivative $\mathrm{dT}_{c} / \mathrm{dP}$ may be an indication that higher values of $\mathrm{T}_{c}$ should be reached through chemical substitution. Whereas this strategy has been successful for most cuprates (one of the most famous example has been given by Bednorz and Müller who induced high $\mathrm{T}_{c}$ below $30 \mathrm{~K}$ in $\mathrm{La}_{2} \mathrm{CuO}_{4}$ by partial substitution of La by Ba [20]), it was also shown that chemical substitution and external hydrostatic pressure may have opposite effects on $\mathrm{T}_{c}$ [21]. On the other hand, our interpretation of chemical substitution effect origin (correlation with the polarization) is quite different from the one usually admit for the superconductors (correlation with the atomic distances [22]), and may constitute an alternative (or additional) explanation.

\section{Acknowledgments}

We wish to acknowledge M. Sieskind for providing us the single crystal of BaFI.
[1] T. J. Wieting and J. L. Verble, in Electrons and phonons in layered crystal structures edited by T. J. Wieting and M. Schlüter (D. Reidel publishing company, Dordrecht, Holland, 1979), Vol. 3.

[2] E. Barrena, S. Kopta, D.F. Ogletree, D.H. Charych and M. Salmeron, Phys. Rev. Lett. 82, 2880 (1999).

[3] J. S. Schilling and S. Klotz, in Properties of HighTemperature Superconductors edited by D. M. Ginsberg (World scientific, Singapore, 1992), Vol. 3

[4] N. Narita, S. Nagai and S. Suzuki, Phys. Rev. B 64, 245408 (2001).

[5] Y.R. Shen, T. Gregorian and W. B. Holzapfel, High Press. Res. 7, 73 (1992).

[6] F. Decremps, M. Fischer, A. Polian, J. P. Itié and M. Sieskind, Phys. Rev. B 59, 4011 (1999).

[7] A. Rulmont, Spectrochem. Acta. 30A, 161 (1974).

[8] M. Sieskind and J. Morel, J. Sol. St. Chem. 144, 339 (1999).

[9] J. F. Scott, J. Chem. Phys. 49, 2766 (1968).

[10] F. Decremps, thèse, Université Paris VI, 1998.

[11] H. P. Beck, J. Solid State Chem. 17, 275 (1976).

[12] Y.R. Shen, U. Englisch, L. Chudinovskikh, F. Porsch, R. Haberkorn, H.P. Beck and W.B Holzapfel, J. Phys.
Condens. Matter, 6, 3197 (1994).

[13] N. Subramanian, N. V. Chandra Shekar, P. Ch. Sahu, Mohammad Yousuf and K. Govinda Rajan, Phys. Rev. B 58, R555 (1998).

[14] M. Sieskind, M. Ayadi and G. Zachmann, Phys. Status Solidi b 136, 489 (1986).

[15] R. Letoullec, J.P. Pinceaux and P. Loubeyre, High Pressure Res. 1, 77 (1988).

[16] G.J. Piermarini, S. Block, J.D. Barnett, and R.A. Forman, J. Appl. Phys. 46, 2774 (1975).

[17] M. Sieskind (unpublished)

[18] G. Kalpana, B. Palanivel and I. B. Shameem Banu, Phys. Rev. B 56, 3532 (1997).

[19] Y.R. Shen, T.Gregorian, W.B. Holzapfel, High pressure Res. 7, 73 (1992).

[20] J. G. Bednorz and K. A. Müller, Z. Phys. B: Condens. Matter 64, 189 (1986).

[21] G. Nieva, S. Ghamaty, B. W. Lee, M. B. Maple and I. K. Schuller, Phys. Rev. B 44, 6999 (1991).

[22] A. A. R. Fernandes, J. Santamaria, S. L. Nakamura, J. Guimpel and I. K. Schuller , Phys. Rev. B 44, 7601 (1991). 